\title{
Observational properties of peculiar core-collapse super- novae producing r-process elements
}

\author{
Tatsuki Hasegawa ${ }^{1, *}$, Masaomi Tanaka ${ }^{1, * *}$, Nobuya Nishimura ${ }^{2,3}$, Kyohei Kawaguchi ${ }^{4}$, \\ Sei Saito ${ }^{1}$, and Nanae Domoto ${ }^{1}$ \\ ${ }^{1}$ Astronomical Institute, Tohoku University, Aoba, Sendai 980-8578, Japan \\ ${ }^{2}$ Astrophysical Big Bang Laboratory, CPR, RIKEN, Wako, Saitama 351-0198, Japan \\ ${ }^{3}$ RIKEN Nishina Center for Accelerator-Based Science, Wako, Saitama 351-0198, Japan \\ ${ }^{4}$ Institute for Cosmic Ray Research, The University of Tokyo, 5-1-5 Kashiwanoha, Kashiwa, Chiba \\ 277-8582, Japan
}

\begin{abstract}
We investigate the effects of r-process elements on the electromagnetic wave emission from peculiar core-collapse supernovae. We perform a series of radiative transfer simulations based on one-dimensional hydrodynamical explosion models. Varying the mass of ${ }^{56} \mathrm{Ni}$ and r-process nuclei, we examine the effects of r-process elements on the light curves. The ${ }^{56} \mathrm{Ni}$-powered explosion models with a large amount of r-process-rich material show redder color because the high opacity of r-process elements suppresses the emission at shorter wavelengths. Comparing the results with the color of observed supernovae, we find that the upper limit for the production of $r$-process elements is $0.1 M_{\odot}$ for ${ }^{56} \mathrm{Ni}$-powered supernovae.
\end{abstract}

\section{Introduction}

The cosmic origin of r-process elements is still under debate. From the recent observations of GW170817 and the relevant emission called kilonova, neutron star mergers are thought to be the plausible candidate. However, it is not yet clear if the neutron star mergers produce the entire r-process elements through the galactic chemical evolution (e.g., [1]). As an alternative scenario, core-collapse supernovae (CCSNe) are regarded as a possible candidate. Typical $\mathrm{CCSNe}$ are thought to explode by the neutrino-heating mechanism, which makes r-process nucleosynthesis difficult due to the decrease of neutron-richness (e.g., [2]). However, in recent studies, peculiar CCSNe such as magneto-rotational SNe (e.g. [3]) and the collapsars with the disk wind (e.g., [4]) are suggested to be able to produce r-process elements. In this study, we perform one-dimensional (1D) hydrodynamical calculations and 1D radiative transfer calculations to understand the possible observational properties of peculiar CCSNe with the production of r-process elements.

\footnotetext{
*e-mail: tatsuki.hasegawa@astr.tohoku.ac.jp

**e-mail: masaomi.tanaka@astr.tohoku.ac.jp
} 


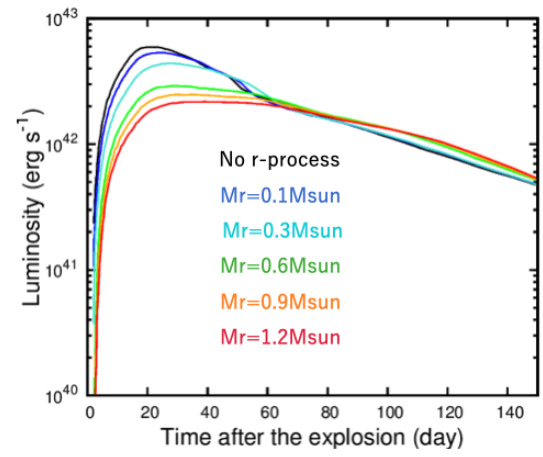

Figure 1. The bolometric luminosities of $\mathrm{SNe}$ with different r-process masses.

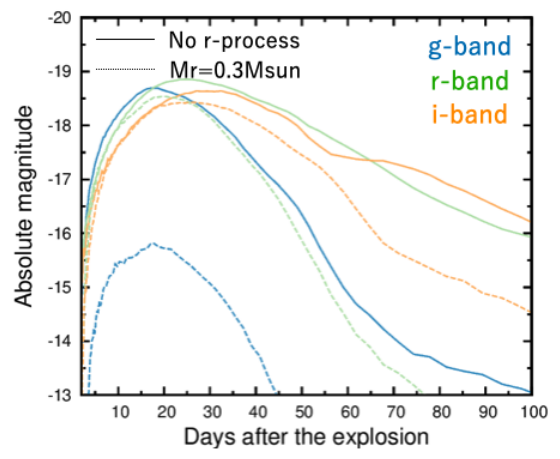

Figure 2. The light curves in each optical band. The solid lines show the model with no r-process elements while the dashed lines show the model with $0.3 M_{\odot}$ of $\mathrm{r}$ process elements.

\section{Method}

We first perform the 1D hydrodynamical and nucleosynthesis calculations using blcode ${ }^{1}$ and nucleosynthesis network with 21 nuclei $^{2}$ to derive the ejecta structure and the composition of the elements. We use a progenitor with the zero-age main sequence (ZAMS) mass of $45 M_{\odot}$ (or $13 M_{\odot}$ just before the collapse, s45 [5]). This star is exploded with the kinetic energy of $10^{52} \mathrm{erg}$, and we track the time evolution of the ejected material. The ${ }^{56} \mathrm{Ni}$ mass in the ejected material is $0.36 M_{\odot}$, which is consistent with ${ }^{56} \mathrm{Ni}$ mass estimated in CCSNe associated with gamma-ray bursts (GRBs).

Using the results of the hydrodynamical calculations, we perform 1D radiative transfer (RT) calculations [6-8]. R-process elements $(Z>30)$ are assumed to be distributed uniformly in the ejecta with the solar r-process abundance pattern. We assume r-process elements are stable as most of the r-process nuclei are close to the stable nuclei in the timescale of interest. Under these assumptions, we calculate the 6 cases with different total mass of r-process elements, i.e., $M_{\mathrm{r}-\text { proc }} / M_{\odot}=0,0.1,0.3,0.6,0.9$, and 1.2 . We do not consider radioactive decay luminosity of r-process elements as it is subdominant compared with that of ${ }^{56} \mathrm{Ni}$ in our models.

\section{Results}

Figure 1 shows the light curves of the bolometric luminosity from 1D RT calculations. The different colors indicate the different masses of $r$-process elements. The model without $r-$ process elements is depicted as the black line. With more r-process elements, the peak luminosity in the bolometric light curve decreases and timescale of the evolution becomes longer.

Figure 2 shows the light curves in the optical band. The solid lines show the model without r-process elements, while the dashed lines show the model with $0.3 M_{\odot}$ of r-process elements. In the model with $0.3 M_{\odot}$ of r-process elements, the light curves in shorter wavelength are remarkably suppressed. The differences in the light curves are attributed to the high opacity of r-process elements, which prevent the photon escaping from the SN ejecta.

\footnotetext{
${ }^{1}$ https://stellarcollapse.org/index.php/SNEC.html

${ }^{2}$ http://cococubed.asu.edu/code_pages/burn_helium.shtml
} 


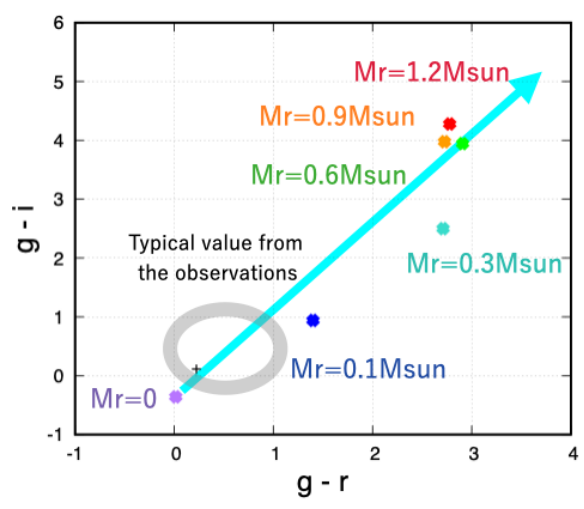

Figure 3. The calculated explosion models on the color-color diagram $(g-i$ vs $g-r)$.

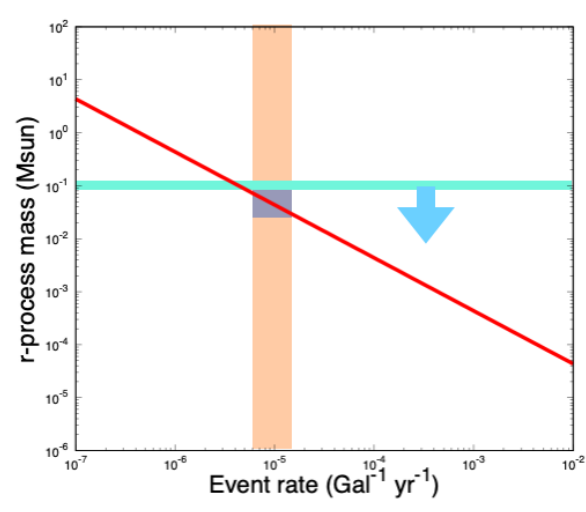

Figure 4. The event rate and the mass of $r-$ process elements produced in one event.

Moreover, the opacity depends on the wavelength, showing a higher opacity toward shorter wavelength [9], which makes the light curve in shorter wavelength more suppressed.

Figure 3 shows the color-color diagram compared with the observational data. The grey region indicates the typical color of observed SNe [10]. From this figure, SNe producing r-process elements larger than $0.1 M_{\odot}$ are deviated from the observed color of typical SNe. This implies that such red SNe have never been observed yet. We can constrain the mass of r-process elements produced in one CCSN, $M_{\mathrm{r}-\text { proc }} \leq 0.1 M_{\odot}$.

\section{Discussion}

We discuss the possibility of peculiar SNe such as magneto-rotational $\mathrm{SNe}$ as the origin of r-process elements. The red line in Figure 4 shows the mass required to explain the total mass of r-process elements in the Galaxy as a function of the event rate [11]. If the event rate of peculiar SNe is assumed to be similar to that of the SNe with GRB (about $10^{-5} \mathrm{Gal}^{-1} \mathrm{yr}^{-1}$ [12]), the required mass of r-process elements per one event to explain the current total mass in the Galaxy is about several $\times 10^{-2} M_{\odot}$. From the result of this paper, it is found that the mass of r-process elements produced by one SN is less than $0.1 M_{\odot}$. Therefore, the possibility that such peculiar SNe produces r-process elements is not rejected from the observation of typical SNe.

\section{References}

[1] Cote, B., Eicher, M. \& Arcones, A., et al. 2019, ApJ, 875, 106

[2] Cowan, J. J., Sneden, C., Lawler, J. E., et al. 2021, Reviews of Modern Physics, 93, 15002

[3] Nishimura, N., Sawai, H. \& Takiwaki, T., et al. 2015, ApJ, 836, L21

[4] Siegel, D. M., Barnes J. \& Metzger, B. D., 2020, Nature, 569, 7755

[5] Sukhbold, T., Ertl, T. \& Woosley, S. E., et al. 2016, ApJ, 821, 38

[6] Tanaka, M. \& Hotokezaka, K., 2013, ApJ, 775, 113

[7] Kawaguchi, K., Shibata, M. \& Tanaka, M., 2018, ApJ, 865, L21

[8] Tanaka, M., Kato, D., Gaigalas, G., et al. 2020, MNRAS, 496, 1369

[9] Tanaka, M., Kato, D., Gaigalas, G., et al. 2018, ApJ, 852, 109 
[10] Stritzinger, M. D., Taddia, F. \& Burns, C. R., et al. 2015, AA, 609, A135

[11] Hotokezaka, K., Beniamini, P. \& Piran, T., 2018, International Journal of Modern Physics D, 27, 1842005

[12] Wanderman, D. \& Piran, T., 2010, MNRAS, 406, 1944 